\title{
Broadband Light Treatment for Multiple Benign Lichenoid Keratoses of Flat Erythematous Type
}

Mi Ryung Roh ${ }^{1}$

Sung Bin $\mathrm{Cho}^{2,3}$

${ }^{1}$ Department of Dermatology, Gangnam
Severance Hospital, Yonsei University College of
Medicine, Seoul, Korea
${ }^{2}$ Department of Dermatology and Cutaneous
Biology Research Center, International St. Mary's
Hospital, Catholic Kwandong University College of
Medicine, Incheon, Korea
${ }^{3}$ Kangskin Sillim Dermatology Clinic, Seoul,
Korea

Received June 13, 2018

Accepted June 18, 2018

Correspondence
Sung Bin Cho
Department of Dermatology and Cutaneous
Biology Research Center, International St. Mary's
Hospital, Catholic Kwandong University College of
Medicine, 25 Simgok-ro, Seo-gu, Incheon 22711,
Korea
Tel.: +82-32-290-3141
Fax: +82-32-290-3142
E-mail: drsbcholagmail.com
(C) Korean Society for Laser Medicine and Surgery
(c) This is an open access article distributed under the
terms of the Creative Commons Attribution Non-
Commercial License (http://creativecommons.org/
licenses/by-nc/4.0) which permits unrestricted non-
commercial use, distribution, and reproduction in any
medium, provided the original work is properly cited.
Benign lichenoid keratosis (BLK) presents as solitary or multiple pinkviolaceous to rusty brown macules or papules on the extremities, chest, and face. Clinically, BLK lesions are categorized into flat erythematous, flat pigmented, and plaque-like types. This paper describes a Korean patient with multiple, early stage, flat erythematous type BLK lesions on the face who was treated effectively and safely with a single session of broadband light (BBL) treatment. The patient underwent one session of a BBL treatment at a fluence of $8-9 \mathrm{~J} / \mathrm{cm}^{2}$ and a pulse duration of $15-20$ msec over multiple passes using $500-n m-$ and $515-n m$ cut-off filters. One month after the BBL treatment, significant improvement in flat erythematous BLK lesions, along with mild hyperpigmentation, was recorded, and the patient was satisfied with the results. Early and noninvasive intervention with BBL energy using proper cut-off filters according to the characteristics of the BLK lesions can effectively reduce inflammatory reactions and prevent disease progression.

\section{Key words}

Benign lichenoid keratosis; Lichen planus-like keratosis; Flat erythematous type; Broadband light; Solar lentigo 


\section{INTRODUCTION}

Benign lichenoid keratosis (BLK) or lichen planus (LP)like keratosis clinically presents as solitary or multiple pink-violaceous to rusty brown macules or papules on the extremities, chest, and face. ${ }^{1-3}$ BLK has been suggested to arise from regressing solar lentigines and reticulated seborrheic keratoses (SKs). ${ }^{4,5}$ However, definite history of preceding lentigines or SKs cannot always be found in all patients with BLK. Thereby, histopathological classifications of BLK include LP-like keratosis, SK-like keratosis, and lupus erythematosus-like keratosis. ${ }^{1}$

Clinically, BLK lesions are categorized into flat erythematous, flat pigmented, and plaque-like types. ${ }^{6}$ Treatment modalities for BLK include excisional biopsy, curettage and electrodessication, cryotherapy, and ablative laser treatments using 2,940-nm erbium (Er):yttriumaluminum-garnet (YAG) or 10,600-nm carbon dioxide (CO2) lasers. Deciding on a particular treatment can be determined according to the clinical types and locations of target lesions and histopathological features thereof. However, the use of ablative treatment systems for treating BLK lesions on the face in Asian patients is limited by a high risk of prolonged postinflammatory erythema and hyperpigmentation. In this report, we describe a Korean patient with multiple, early stage, flat erythematous type BLK lesions on the face that were effectively and safely treated with a single session of broadband light (BBL) treatment.

\section{CASE REPORT}

A 73-year-old Korean female visited our clinic presenting with asymptomatic, multiple, flat, and erythematous lesions on the nose, forehead, and cheeks that had persisted over 1 month. She had no pertinent family history or medical history, except for primary hypertension. The patient had been treated with oral and topical medications at other clinics, the clinical outcomes of which were unsatisfactory. She had no pertinent history of light- or laser-based treatments on the face within the last three months.

On her first visitation, multiple, well-demarcated, flat, erythematous maculopapules and a few brownish lentiginous lesions were noted (Fig. 1A). Photographs were taken under normal, polarized, and ultraviolet light exposures using an imaging tool (Janus ${ }^{\circledR}$; PSI Corporation, Ltd., Seoul, Koreal at baseline to accentuate skin pigmentation and vascularity. Photographs taken under polarized light exhibited well-accentuated erythematous lesions with high vascularity (Fig. 2A). Under ultraviolet light, however, the erythematous lesions were unremarkable (Fig. 3A). Meanwhile, the brownish lentiginous lesions were unremarkable under the polarized light and accentuated under the ultraviolet light. Accordingly, the patient was clinically diagnosed with flat erythematous type BLK (Fig. 1A).

After obtaining written informed consent, the patient was treated with one session of BBL treatment ISciton Joule Platform using the BBL module; Sciton, Palo Alto, CA, USA) for multiple BLKs on the face. The face was gently cleansed with a mild soap and pretreated with topical anesthetic cream leutectic mixture of $2.5 \%$ lidocaine $\mathrm{HCl}$ and $2.5 \%$ prilocaine, EMLA; Astra Pharmaceuticals, Westborough, MA, USA) for 1 hour. After applying chilled ultrasonic gel, BBL energy was delivered with a cut-off filter of $515 \mathrm{~nm}$, a fluence of $9 \mathrm{~J} / \mathrm{cm}^{2}$, a pulse duration of $15 \mathrm{msec}$, and a cooling crystal temperature of $15^{\circ} \mathrm{C}$ : two passes of BBL treatment were delivered on both cheeks with a spot size of $15 \mathrm{~mm} \times 45 \mathrm{~mm}$ and on the forehead, nose, and perioral and periorbital areas with a spot size of $15 \mathrm{~mm} \times 15 \mathrm{~mm}$ using a square adaptor (Sciton). Then, the flat erythematous BLK lesions were treated with an additional two passes at a fluence of $8 \mathrm{~J} / \mathrm{cm}^{2}$, a pulse
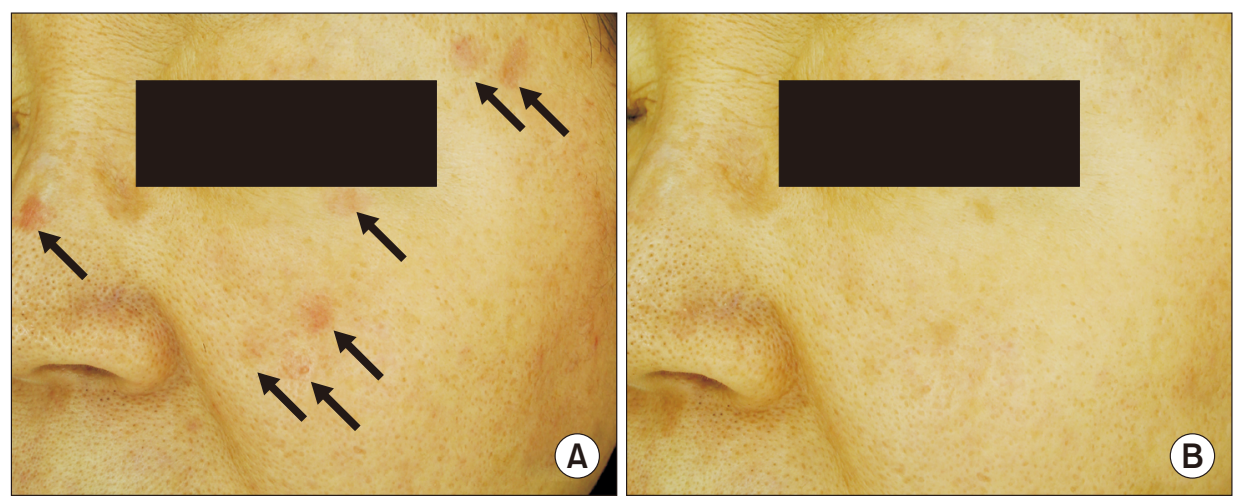

Fig. 1. Photographs of a 73-year-old female patient. The patient was treated with a single session of broadband light (BBL) treatment for multiple, flat erythematous benign lichenoid keratoses (BLK) on the face (arrows). (A) Baseline and (B) one month after BBL treatment. Normal light exposure. Left oblique view. 

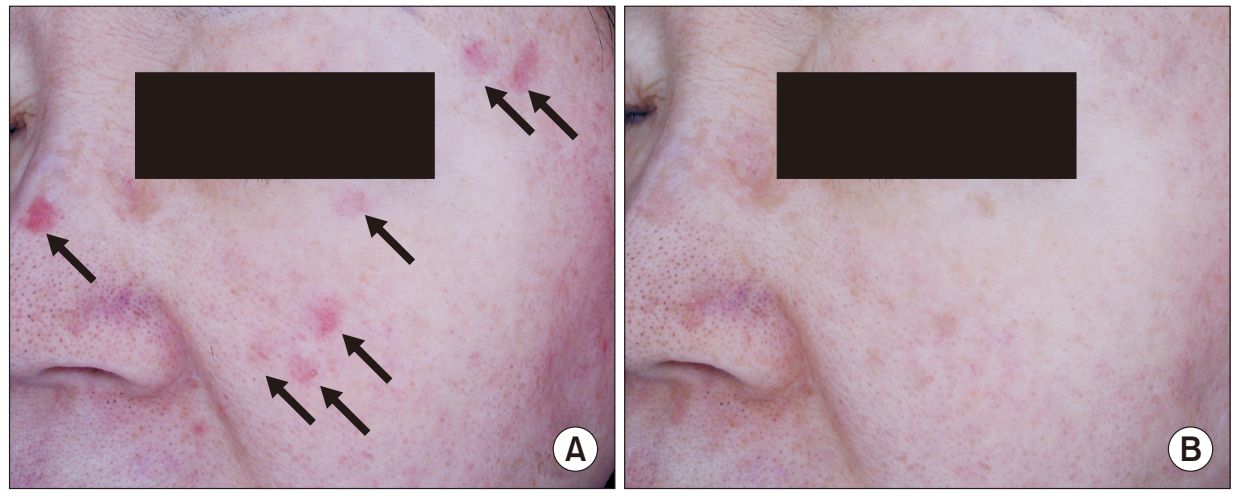

Fig. 2. Photographs taken under polarized light exposure. BLK lesions (arrows) at (A) baseline and (B) one month after BBL treatment. Left oblique view.
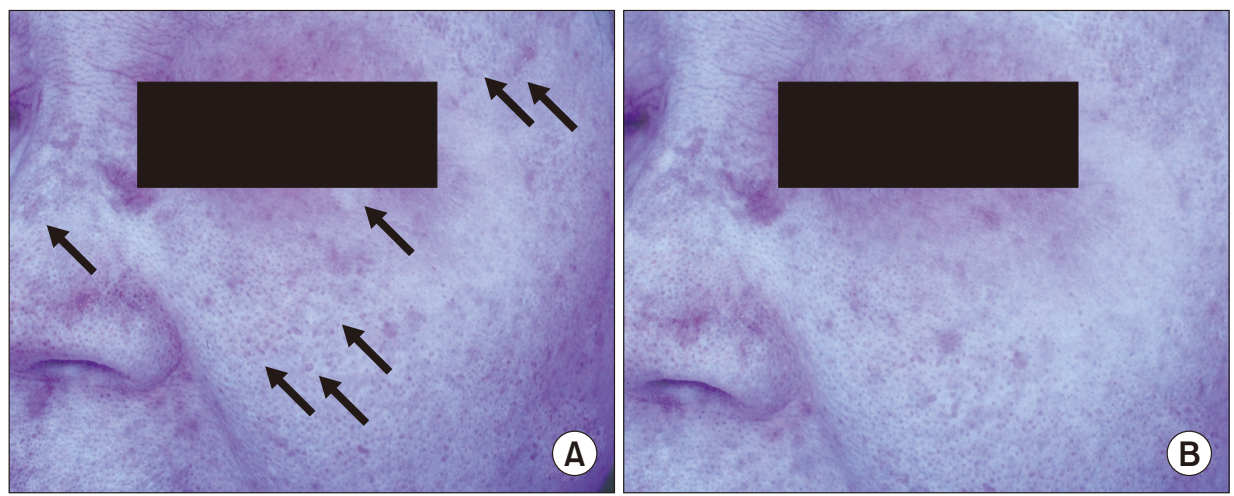

Fig. 3. Photographs taken under ultraviolet light exposure. BLK lesions (arrows) at (A) baseline and (B) one month after BBL treatment. Left oblique view.

duration of $20 \mathrm{msec}$, and a cooling crystal temperature of $15^{\circ} \mathrm{C}$ using a 500PB-cut-off filter (Sciton) and a 7-mm round adaptor (Sciton). After BBL treatment, the treated areas were cooled with icepacks. No prophylactic systemic or topical antibiotics, antivirals, or corticosteroids were prescribed. The patient was recommended to apply sunscreen thereafter.

One month after the BBL treatment, the flat erythematous BLK lesions had markedly improved with mild hyperpigmentation, and the patient was satisfied with the results (Fig. 1B). Post-treatment photographs under polarized light also exhibited remarkable reductions in inflammatory lesions and associated erythema (Fig. 2B). Nonetheless, post-treatment dyspigmentation, which was suggestive of BLK-associated lentiginous lesions or postinflammatory hyperpigmentation, was visible under ultraviolet light (Fig. 3B). Pain during the treatment was tolerable and no remarkable major side effects, including burn, crusts, oozing, petechiae or bruising, bleeding, edema, prolonged erythema, and atrophic or hypertrophic scarring, were encountered.

\section{DISCUSSION}

In this report, we describe the use of pulsed delivery of
BBL energy to non-invasively and effectively treat multiple, early stage, flat erythematous type BLK lesions on the face. Histopathologic evaluation to diagnose BLK is usually unnecessary, except for highly suspicious lesions that should be differentiated from malignant conditions. In our patient, the diagnosis of BLK of flat erythematous type was clinically made according to the patient's history of skin lesions and the evaluation of photographs taken under normal, polarized, and ultraviolet light exposures. Although the flat erythematous lesions in our patient were unremarkable under ultraviolet light exposure, post-treatment photographs revealed lightly brownish lentiginous changes. We suggest that lentiginous lesions could have developed in the early stage BLK lesions in our patient, which lacked noticeable pigmentation components, after the resolution of inflammatory reactions by $B B L$ treatment or that post-BBL dyschromia could have resulted from postinflammatory hyperpigmentation. Nonetheless, we propose that early intervention with BBL energy using 515- and 500PB-cut-off filters can effectively treat the inflammatory reactions of BLK lesions and safely prevent disease progression.

Broadband light treatment has been effectively applied for the treatment of various inflammatory skin lesions, including atopic dermatitis and acne vulgaris. ${ }^{7-9} \mathrm{BBL}$ en- 
ergy with the cut-off wavelength of $590 \mathrm{~nm}$ can decrease inflammatory cell infiltration in the dermis, as the laser pulses from 595-nm pulsed dye lasers target dermal microvascular components. ${ }^{7-9}$ Theoretically, laser or light energy sources at wavelengths ranging from $400 \mathrm{~nm}$ to $600 \mathrm{~nm}$ are more readily absorbed by oxyhemoglobin and melanin, compared to those at longer wavelengths over $600 \mathrm{~nm} .{ }^{10}$ However, the risk of significant vascular damage and associated side effects, particularly in Asian patients, is higher at wavelengths from $400 \mathrm{~nm}$ to $600 \mathrm{~nm}$, compared to wavelengths over $600 \mathrm{~nm}$.

In this report, we treated our patient with BBL treatment combining the use of 515- and 500PB-cut-off filters, in addition to the use of a relatively low fluence (8-9 J/ $\mathrm{cm}^{2}$ ) and long pulse duration (15-20 msec). Therewith, additional pulses with the 500PB-cut-off filter could be safely delivered to lesions pretreated with 515-nm BBL at a tolerable pain level. Moreover, we believe that the noninvasive characteristics of $\mathrm{BBL}$ treatment significantly reduced the risks of major side effects that are common during the treatment of various types lesions on the face in Asian patients, compared to the ablative treatment systems, including electrosurgery, CO2 laser, and Er:YAG laser. Post-BBL oral or topical medications and occlusive wound care was unnecessary, except for the use of emollient and sunscreen.

In conclusion, we were able to effectively and safely treat a Korean patient with multiple, early stage, flat erythematous BLK lesions with BBL energy. Our patient experienced noticeable clinical improvements in inflammatory lesions without major side effects. We suggest that early and non-invasive intervention with BBL energy using proper cut-off filters according to the characteristics of BLK lesions can effectively reduce inflammatory reactions therein and prevent disease progression. Nonetheless, additional controlled clinical trials with histopathological evaluation are needed to confirm our findings.

\section{ACKNOWLEDGEMENTS}

We would like to thank Duke Song (Consultant; Sciton, Palo Alto, CA, USAl for his assistance with technical support. We would also like to thank Anthony Thomas Milliken, ELS (Editing Synthase, Seoul, Koreal for his help with the editing of this manuscript.

\section{REFERENCES}

1. Jang KA, Kim SH, Choi JH, Sung KJ, Moon KC, Koh JK. Lichenoid keratosis: a clinicopathologic study of 17 patients. J Am Acad Dermatol 2000;43:511-6.

2. Barranco VP. Multiple benign lichenoid keratoses simulating photodermatoses: evolution from senile lentigines and their spontaneous regression. J Am Acad Dermatol 1985;13:201-6.

3. Abdulla FR, Mutasim DF. Multiple benign lichenoid keratoses. J Am Acad Dermatol 2010;62:900-1.

4. Mehregan AH. Lentigo senilis and its evolution. J Invest Dermatol 1975;65:429-33

5. Berman A, Herszenson S, Winkelmann RK. The involuting lichenoid plaque. Arch Dermatol 1982;118:93-6.

6. Bugatti L, Filosa G. Dermoscopy of lichen planus-like keratosis: a model of inflammatory regression. J Eur Acad Dermatol Venereol 2007;21:1392-7.

7. Oh SH, Bae BK, Kim TG, Kwon YS, Lee JH, Lee KH. Effective treatment of facial redness caused by atopic dermatitis using intense pulsed light systems. Dermatol Surg 2010;36:475-82.

8. Omi T, Bjerring P, Sato S, Kawana S, Hankins RW, Honda M. $420 \mathrm{~nm}$ intense continuous light therapy for acne. J Cosmet Laser Ther 2004;6:156-62.

9. Ito Y, Cho SB. Modified 3-step broadband light treatment for inflammatory acne vulgaris and post-acne erythema in Asian patients. Med Lasers 2017;6:102-6.

10. Gupta AK, Gover MD, Nouri K, Taylor S. The treatment of melasma: a review of clinical trials. J Am Acad Dermatol 2006;55:1048-65. 